\title{
Jerarquizar la extensión universitaria desde la escritura académica. 10 años de +E: Revista de Extensión Universitaria
}

\author{
Cecilia lucci \\ Universidad Nacional del Litoral, Argentina \\ ciucci@unl.edu.ar \\ (iD) orcid.org/0000-0003-0073-2124
}

+E nació en noviembre de 2011 en el marco del XI Congreso lberoamericano de Extensión Universitaria de la Unión Latinoamericana de Extensión Universitaria (ULEU) que se desarrolló en la Universidad Nacional del Litoral (UNL).

Desde el inicio, la revista se propuso constituir un espacio de debate y reflexión teórica, epistemológica y política sobre la forma de pensar y hacer extensión en Latinoamérica y el Caribe. Con la misma intensidad se planteó asociar la escritura académica a la función social desde la rigurosidad conceptual y argumentativa, que es una de las formas de jerarquizarla.

Históricamente, y hasta comienzos de este siglo, la extensión universitaria quedó relegada de la escritura académica por ser considerada, en términos generales, una función menor, de carácter volitivo, disociada la mayoría veces de las trayectorias curriculares y de la producción de conocimientos. En el mejor de los casos, desde la extensión se podía asumir prácticas de escritura relacionadas con la divulgación científica (cuestión ciertamente importante para las universidades).

A partir de una serie de cambios a nivel global y de la propia cultura académica (especialmente vinculados a la concepción del conocimiento y a los modos de abordajes de problemáticas sociales complejas), en los últimos 20 años se fue consolidando en el discurso universitario latinoamericano el espacio de la extensión universitaria, se la fue resignificando de los postulados reformistas que le dieron origen. Se la fue articulando a perspectivas teóricas, a escuelas de pensamiento, a determinados referentes y metodologías que le permitieran leer y actuar respecto del rol de la universidad en la comunidad. A su vez, desde la extensión se fue traccionando, cada vez con mayor fuerza, la necesidad de integrar las funciones universitarias —docencia, investigación, extensión- para marcar la pertinencia social del tipo de conocimiento que se imparte en las aulas, las agendas de investigación y las intervenciones que requiere la comunidad. Diálogo, comunicación, escucha, transformación, enseñanzas y aprendizajes compartidos, son algunos de los significantes más pronunciados.

Es aun más reciente la problematización de la extensión como campo de estudio, como objeto de análisis. ${ }^{1}$ Esto es, la posibilidad de contar con producciones en donde se pueda expresar ese andamiaje conceptual, el entramado teórico y metodológico y las definiciones políticas que determinan las razones, los temas, los sujetos y los modos en los que las universidades abordan la misión social. En este sentido, dos hechos significativos marcan esta tendencia. Por una parte, la relativamente reciente constitución de redes universitarias de

1) Un dato que apoya esta tesis es que, en Argentina, fue recién en el VIII Encuentro Nacional y el V Latinoamericano La Universidad como objeto de investigación que tuvo lugar en la UNL en 2017 que apareció por primera vez la extensión universitaria como eje del Encuentro. El I Encuentro se hizo en la Universidad de Buenos Aires en 1995 y en estos 22 años aparecieron ejes como universidad y sociedad de manera ambigua o vinculación tecnológica pero nunca un eje específico sobre extensión universitaria 
extensión a nivel de los países y de las regiones ${ }^{2}$ ha sido un motor importante no solo para la construcción de agendas de trabajo compartidas sino para dar cuenta de la pluralidad de perspectivas sobre la extensión. Por otra parte, la aparición de revistas académicas específicas -el promedio de edad de las revistas no supera los ocho años- ha destacado a esta función sustantiva como parte estructurante de distintos modelos universitarios. Modelos en los que se ponen en juego el papel social que cumple el conocimiento científico y la contribución de las universidades al territorio (Tommasino y Cano, 2016; Cano, 2017; Menéndez, 2017; Stein, 2018).

Es en diálogo con este contexto que surge y se despliega $+\mathrm{E}$.

\section{El perfil editorial de $+\mathbf{E}$}

La propuesta editorial se inscribió en la política de comunicación y de internacionalización de extensión universitaria de la UNL y se sustentó en tres principios básicos: diversidad de perspectivas, calidad y pertinencia de los escritos y acceso libre a la publicación. Estos principios cruzan hoy en día la política editorial y se traducen en instrumentos para llevarlos adelante. Así:

1) La diversidad de perspectivas está dada en la apertura del juego a todo el sistema universitario latinoamericano y caribeño, poniendo de relieve enfoques, metodologías y trayectorias de las universidades, especialmente las públicas.

2) La calidad y la pertinencia de los escritos requiere esfuerzos en tres sentidos. Por un lado, en la elección del campo problemático o el eje de gestión que va a abordar cada número. Por otro, en cuanto a centrar la atención en los mecanismos de selección de los escritos a partir de un proceso de evaluación bajo el sistema "doble ciego", en donde el revisor respeta el punto de vista del autor. En un tercer sentido, respecto de procurar escritos que sean rigurosos, críticos de los procesos, que presenten actualidad en las discusiones y que incorporen la mirada (o la autoría) con los sujetos no universitarios con quienes se construyen las intervenciones.

3) El acceso libre a la publicación va mucho más allá que adherir a los postulados de la Política de Acceso Abierto, aboga por la ciencia abierta y por su correlato con la ciencia ciudadana como premisa ética y política para pensar y actuar desde la universidad.

+E se estructura en secciones que dan lugar a discusiones desde las más generales, sobre el tema que aborda el número de la revista, hasta los análisis más particulares de las intervenciones (Ver Cuadro 1).

En la revista pueden escribir docentes, investigadores, estudiantes, referentes sociales y de organizaciones sociales, así como funcionarios públicos que estén vinculados a líneas de extensión.

Entre las exigencias de escritura cabe señalar algunas condiciones mínimas en donde deben estar claros los enfoques de extensión y de la problemática que se aborda, la situación previa a la intervención, y un análisis crítico sobre los procesos y los aportes (que involucren tanto a los actores universitarios como a los no universitarios). 
Cuadro 1: Secciones y detalles de escritos que involucra

\begin{tabular}{ll}
\hline Perspectivas & - Panoramas de las discusiones teóricas, metodológicas o políticas actuales re- \\
& specto de conceptos o categorías del tema central de la convocatoria. Referencias a \\
& los aportes de determinados autores o escuelas de pensamiento que interpelen a la \\
& misión social de la universidad. \\
& - Recorridos históricos o estudios comparativos que permitan dar cuenta de un \\
& "estado de situación" en un determinado país, región o institución en cuanto a pro- \\
& cesos y actores sociales vinculados al tema central. \\
& - Abordajes críticos a partir de un estudio de caso que permitan poner en discusión \\
& enfoques teóricos, conceptuales y metodológicos con relación a la temática central. \\
& - Análisis sobre el papel de las instituciones de educación superior, las políticas \\
& públicas y la incidencia social que tiene el tema central. \\
\hline Desafíos de gestión & - Estrategias de la gestión universitaria con referencia al tema central de la convo- \\
& catoria. Se deben exponer los enfoques, conflictos, desafíos y limitaciones en el \\
diseño e implementación de las políticas universitarias respecto de la temática que \\
aborda la revista. Las experiencias deben estar avanzadas.
\end{tabular}

Fuente: elaboración propia.

\section{Trayectoria de $+\mathrm{E}$}

Hoy $+E$ constituye una referencia ineludible en materia de extensión en la región. Llevamos 10 convocatorias; 13 números editados (8 ediciones impresas y 13 ediciones digitales); 252 artículos publicados (sobre más de 470 presentados); más de 100 revisores procedentes de distintas disciplinas y más de una decena de artículos con más de 1000 descargas (el artículo que más descarga tiene está en las 18.000). Ver Cuadro 2.

+E está incorporada a 22 sitios entre Catálogos, Bases de Datos y Directorios. Forma parte de 4 portales y tiene 13 declaraciones de interés que abarcan redes de extensión, grupos de investigación, una Cátedra UNESCO, entre otras instituciones. El hecho de estar incluida en Núcleo Básico de Revistas Científicas Argentinas, en CAPES de Brasil, en Erihplus, Latindex 2.0; DOAJ; Dialnet, entre otros sitios, la ha posicionado en el nivel 1 de revistas académicas.

En marzo de 2020, se publicó el Informe 2011-2020 por los 10 años de +E (lucci, 2020) en el que se presentaron aspectos cuantitativos recabados de las métricas que ofrecen el Open Jounal System, el Google scholar y Crossref Matomo. Allí se da cuenta de la procedencia institucional de los autores, los artículos con más de 1000 descargas, el impacto de cita, los 5 artículos más citados, el resumen de visitas a la revista, la cantidad y origen de visitantes, un mapa de visitantes, los sitios de Internet desde donde se accede, el listado de indizaciones, directorios, bases de datos y portales y las declaraciones de interés que tiene la revista. 
Cuadro 2: Números editados

\begin{tabular}{|c|c|c|c|}
\hline Año & \# & Portada & Tema \\
\hline 2011 & 1 & & $\begin{array}{l}\text { Inclusión y cohesión social. Aportes al debate en el marco del XI Congreso } \\
\text { Iberoamericano de Extensión Universitaria. }\end{array}$ \\
\hline 2012 & 2 & & Construir ciudadanía. Debates, desafíos y propuestas de extensión universitaria. \\
\hline 2013 & 3 & & Desarrollo local y regional. Los aportes de extensión a las políticas públicas. \\
\hline 2014 & 4 & & $\begin{array}{l}\text { Universidad y salud. La incidencia de las prácticas de extensión sobre los } \\
\text { determinantes sociales. }\end{array}$ \\
\hline 2015 & 5 & & $\begin{array}{l}\text { Economía social y solidaria. Perspectivas, aportes y desafíos para construir otra } \\
\text { economía. }\end{array}$ \\
\hline 2016 & 6 & & $\begin{array}{l}\text { Integración de la docencia y la extensión. Un desafío para el curriculo } \\
\text { universitario. }\end{array}$ \\
\hline 2017 & 7 & & $\begin{array}{l}\text { Extensión universitaria: agendas, debates y desafíos a } 100 \text { años de la Reforma } \\
\text { de } 1918 .\end{array}$ \\
\hline 2018 & $8 / 9$ & & $\begin{array}{l}\text { Investigación y extensión universitaria. La misión social como campo de } \\
\text { estudio. }\end{array}$ \\
\hline 2019 & $10 / 11$ & & $\begin{array}{l}\text { Cultura(s) en clave de extensión universitaria. Tramas, identidades y políticas en } \\
\text { la construcción del territorio. }\end{array}$ \\
\hline 2020 & $12 / 13$ & & Sujetos y relaciones en extensión universitaria. \\
\hline
\end{tabular}

Fuente: elaboración propia.

Se puede deducir la circulación de +E por considerar, al menos, tres variables: por la procedencia de los autores, los lugares de descargas y las visitas registradas en los artículos y a la revista.

Respecto de la procedencia de los autores, se identifican 15 países: Argentina, Bolivia, Brasil, Canadá, Chile, Colombia, Costa Rica, Cuba, Ecuador, Italia, México, Paraguay, Suecia, Uruguay y Venezuela.

En el Cuadro 3 y en la Figura 1 se identifican la cantidad y el origen de los visitantes así como el mapa de visitas. 


\section{Cuadro 3: Cantidad y origen de los visitantes}

\begin{tabular}{|c|c|c|}
\hline \multicolumn{2}{|l|}{ Origen } & \multirow{2}{*}{$\frac{\text { Visitas }}{32.115}$} \\
\hline \multirow[t]{8}{*}{ Continentes } & Sudamérica & \\
\hline & América del Norte & 2.385 \\
\hline & Europa & 1.326 \\
\hline & Centroamérica & 1.306 \\
\hline & Asia & 78 \\
\hline & África & 69 \\
\hline & Sin referencias & 68 \\
\hline & Oceanía & 10 \\
\hline \multirow[t]{7}{*}{ Países } & Argentina & 23.178 \\
\hline & (2) Brasil & 2.567 \\
\hline & 藤 Uruguay & 2.340 \\
\hline & Colombia & 1.703 \\
\hline & Estados Unidos & 1.313 \\
\hline & - México & 1.047 \\
\hline & España & 913 \\
\hline
\end{tabular}

Fuente: MATOMO. Datos desde el 10 de junio de 2017 al 6 de diciembre de 2020.

Figura 1: Mapa de visitantes

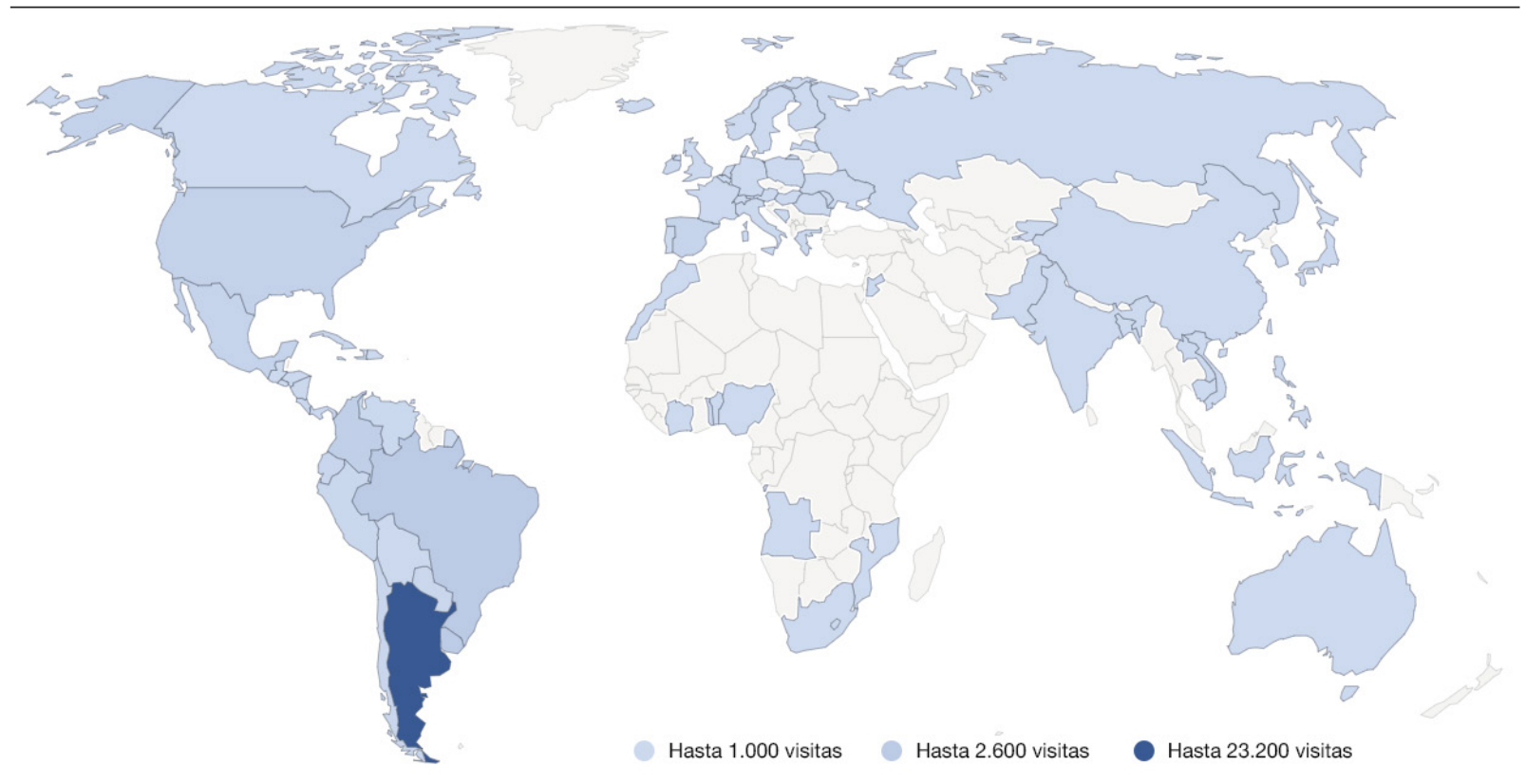

Fuente: MATOMO. Datos desde el 10 de junio de 2017 al 6 de diciembre de 2020 


\section{Red de Editores de Revistas de Extensión de Latinoamérica y el Caribe}

En septiembre de este año, +E impulsó la creación de esta Red de Editores de Revistas de Extensión Universitaria (REDREU) en el marco de la Comisión Permanente de Extensión Universitaria de la Asociación de Universidades del Grupo Montevideo (AUGM). Cooperación y trabajo en red fueron las premisas que orientaron esta propuesta en contraposición a competencia y trabajo individual, que son las que guían a la mayoría de las revistas académicas. Cooperación y trabajo en red también son condiciones que plantea la extensión universitaria.

Tal como lo estipula el documento de creación de la Red (AUGM, 2020), se buscó involucrar a las revistas de extensión universitaria que deseen sumarse a un trabajo colaborativo a partir de reconocer que las revistas:

- son espacios relevantes para dar cuenta de los debates sobre las definiciones (teóricas, epistemológicas, metodológicas), visiones, políticas e ideologías que sustentan a la extensión universitaria;

- recogen la incidencia que producen las acciones tanto para los actores sociales como para la propia universidad y que en muchas de ellas recuperan las voces de las comunidades con las que se trabaja;

- interpelan, desde muchos de los contenidos publicados, al papel social de la universidad, a la formación de los estudiantes, a las estrategias de enseñanza, a la diversidad de aprendizajes, a las agendas de investigación, a los modos en los que se construye y circula en conocimiento, a las maneras en que la universidad actúa frente a las problemáticas sociales vinculadas al entorno; $y$

- son (y deben ser) parte activa de los movimientos por la ciencia abierta y el acceso abierto.

Participan de la Red 35 revistas procedentes de Angola, Argentina, Brasil, Costa Rica, Ecuador, España, Honduras, Nicaragua y Uruguay. A partir de un relevamiento exhaustivo sobre los perfiles, necesidades e intereses de las revistas, se está elaborando el plan de trabajo 2021-2021 que involucrará espacios de capacitación, de discusión y trabajo sobre líneas comunes.

\section{Aportes y desafíos de +E para pensar la universidad y la escritura vinculada a la extensión}

La revista es un espacio habilitante para pensar la universidad, las dinámicas territoriales, los movimientos sociales y las organizaciones, las políticas públicas, la democracia, el poder, los discursos hegemónicos y el lugar del conocimiento científico en esos discursos. +E pone en visibilidad estos tópicos a través de los artículos pero también desde otras instancias y soportes: conversatorios, cursos, entrevistas, presentaciones. La virtualidad permitió que más de 1500 personas procedentes de Argentina, Bolivia, Brasil, Colombia, Costa Rica, Cuba, Ecuador, Honduras, Nicaragua, Paraguay, Perú, Uruguay y Venezuela discutieran sobre metodologías, territorio, multiculturalidad, escritura académica y estrategias de las universidades frente a la pandemia.

Sin perder de vista las particularidades de las intervenciones sociales, hay un esfuerzo de la revista por evitar que se convierta en un reducto de "extensionistas". Se aspira a ser una referencia de lectura, producción y discusión no solo para quienes hacen extensión (univer- 
sitarios y no universitarios), sino para quienes construyen la universidad: docentes, investigadores, estudiantes, graduados, personal de gestión, personal administrativo. También se dirige a los funcionarios públicos que son quienes deciden cómo llevar adelante políticas públicas que afectan el destino de toda una comunidad.

Por tanto, conocer qué tipos de escritos y quiénes son los lectores — vinculados no ya a una revista sino al universo de publicaciones de extensión- se convirtió en una preocupación, y en este sentido las métricas no son suficientes. Así que se lanzó un cuestionario en el mes de noviembre sobre "Escrituras, referencias y lecturas vinculadas a la extensión universitaria" que apunta a recabar información sobre cuáles son las lecturas y categorías teóricas que se valoran al momento de reflexionar y actuar en extensión universitaria, cuáles son considerados los autores de referencia y los sitios en los que publicamos y consultamos. La encuesta está dirigida a la comunidad académica, es anónima, y los resultados serán publicados periódicamente.

Respecto de los artículos, +E pone en crisis los cánones de escritura científica que son utilizados, acríticamente, en publicaciones de congresos y revistas de extensión. Modelo de escritura vinculado a poderosos dispositivos de producción, circulación, control y estandarización (del conocimiento científico y de los modos de escribirlo) de impacto global. Así, pensar el tipo de narrativas más adecuadas a la extensión universitaria también es una de las preocupaciones centrales de la revista. Entender los distintos subgéneros, cruzar los enfoques de extensión y los estilos narrativos son algunas cuestiones que se problematizan en las instancias de capacitación sobre escritura académica que propone periódicamente la revista.

Los desafíos que tiene hoy +E son los mismos que presenta la extensión universitaria y, por ende, la universidad pública: se vuelve imperioso proyectar las discusiones en torno a qué universidad necesitamos, para quiénes las imaginamos, qué valores rectores las van a orientar, para qué modelos de desarrollo y democracia las proponemos y quiénes deben participar de ese debate. En esas discusiones, las revistas de extensión pueden hacer aportes significativos.

\section{Referencias bibliográficas}

AUGM (2020). Documento preliminar de la creación Red de Editores de Revistas de Extensión Universitaria (REDREU) de AUGM. Mimeo.

Cano Menoni, A. (2017). La extensión universitaria y la universidad latinoamericana: hacia un nuevo "orden de anticipación" a 100 años de la revuelta estudiantil de Córdoba. +E: Revista de Extensión Universitaria, 7(7), 6-23. https://doi.org/10.14409/extension.v0i7.7047

lucci, C. (2020). +E: Revista de Extensión Universitaria. Informe 2011-2020. https://hdl.handle.net/11185/5577 Menéndez, G. (2017). Resignificación de la extensión a 100 años de la Reforma Universitaria de 1918. +E: Revista de Extensión Universitaria, 7(7), 24-37. https://doi.org/10.14409/extension.v0i7.7048

Tommasino, H.; Cano, A. (2016). Modelos de extensión universitaria en las universidades latinoamericanas en el siglo XXI: tendencias y controversias. Universidades, (67), 7-24 Unión de Universidades de América Latina y el Caribe Distrito Federal.

Stein, N. (2018). La extensión-comunicación universitaria disputando el lugar de la investigación como fuente de conocimiento. Un recorrido histórico hasta los enfoques actuales. +E: Revista de Extensión Universitaria, 8(9), 14-37. https://doi.org/10.14409/extension.v8i9.Jul-Dic.7866 